\title{
Asymptotic behavior of solutions for the time-delayed equations of Benjamin-Bona-Mahony's type
}

Feng Wei ${ }^{1}$, Pu Zhilin² and Zhu Chaosheng ${ }^{3^{*}}$

*Correspondence: zcs@swu.edu.cn ${ }^{3}$ School of Mathematics and Statistics, Southwest University, Chongqing, 400715, China Full list of author information is available at the end of the article

\begin{abstract}
In this paper, we investigate the asymptotic behavior of the solutions for the equations of Benjamin-Bona-Mahony's type with a time delay. We prove the global existence of solutions and energy decay. By using the Liapunov function method, we shall show that the solution is exponentially decay if the delay parameter $\tau$ is sufficiently small.
\end{abstract}

MSC: 35R10; 35B35; 35Q53

Keywords: Benjamin-Bona-Mahony equation; time delay; exponential decay; Liapunov function

\section{Introduction}

In this paper, we consider a family of dispersive equations of Benjamin-Bona-Mahony's type under the effect of dissipation, and we will investigate the asymptotic behavior of the solutions. Our model can be written in the abstract form

$$
\begin{aligned}
& M u_{t}(x, t)+\alpha L u(x, t)+u(x, t-\tau) u_{x}(x, t)=0, \\
& u(x, t)=u(x+1, t), \\
& u(x, s)=u_{0}(x, s),
\end{aligned}
$$

where $x \in \mathbb{R}^{1}, t>0,-\tau \leq s \leq 0, \alpha>0$, and we set $\Omega=(0,1)$. The operators $M$ and $L$ can be differential operators or pseudo-differential operators, and the orders of pseudodifferential operators $M$ and $L$ are $\mu$ and $s$ with $s \geq \mu \geq 2$.

In the simplest case, when $M$ and $L$ are the differential operators $M=I-\frac{\partial}{\partial x^{2}}, L=-\frac{\partial}{\partial x^{2}}$, (1.1) is the well-known Benjamin-Bona-Mahony model [1]:

$$
u_{t}(x, t)-u_{x x t}(x, t)-\alpha u_{x x}(x, t)+u(x, t) u_{x}(x, t)=0
$$

which describes the unidirectional propagation of weakly nonlinear dispersive long waves where Burger's type dissipation is considered. The existence of global solutions and asymptotic behavior in time have been studied by several authors. The asymptotic behavior of solutions to the generalized Korteweg-de Vries-Burgers and Benjamin-BonaMahony-Burgers equations in one space dimension was studied by Amick, Bona and

(C) 2015 Wei et al. This article is distributed under the terms of the Creative Commons Attribution 4.0 International License (http://creativecommons.org/licenses/by/4.0/), which permits unrestricted use, distribution, and reproduction in any medium, provided you give appropriate credit to the original author(s) and the source, provide a link to the Creative Commons license, and indicate if changes were made. 
Schonbek in [2], by Wang and Yang in [3], and by Bona and Luo in [4]. These results were generalized by Zhang [5] to multiple spatial dimensions. In [6-8] the authors considered a family of equations of KdV and BBM's type described by pseudo-differential operators, and studied the asymptotic behavior in one space dimension. In [9] study the Cauchy problem for a class of nonlinear dissipative equations of Benjamin-Bona-Mahony's type and discuss the existence of a global attractor and estimate its Hausdorff and fractal dimensions. But few of the equations involving delay.

To explain our motivation of introducing a time delay into Benjamin-Bona-Mahony equation, we consider the rate of change of $u$, which we denote by $\frac{D u}{D t}$, is

$$
\begin{aligned}
\frac{D u}{D t} & =\frac{d}{d t} u[x(t), t]=\frac{\partial}{\partial t} u(x, t)+\frac{d x(t)}{d t} \frac{\partial}{\partial x} u(x, t) \\
& =\frac{\partial}{\partial t} u(x, t)+u(x, t) \frac{\partial}{\partial x} u(x, t),
\end{aligned}
$$

where $x(t)$ is understood to change with time at $u=\frac{d x}{d t}$. However, we might have a delay $\tau$ to $u$. In this case the rate of change of $u$ with the delay $\tau$ should be

$$
\begin{aligned}
\frac{D u}{D t} & =\frac{d}{d t} u[x(t-\tau), t]=\frac{\partial}{\partial t} u(x, t)+\frac{d x(t-\tau)}{d t} \frac{\partial}{\partial x} u(x, t) \\
& =\frac{\partial}{\partial t} u(x, t)+u(x, t-\tau) \frac{\partial}{\partial x} u(x, t) .
\end{aligned}
$$

This clearly shows how we obtain the time-delay term $u(x, t-\tau) u_{x}(x, t)$ in Benjamin-BonaMahony equation (1.4). There is literature about delay reaction-diffusion equations [1012], on which our work is based. Recently, the asymptotic behavior of solutions of timedelayed Burgers equation was studied by Liu in [13]. Subsequently, exponentially decay rate of solutions for Benjamin-Bona-Mahony equation (1.4) with a time decay was studied in [14], and in [15] author have obtained the exponentially decay rate of solutions for the time-Delayed Kuramoto-Sivashinsky equation. Here, by using the Liapunov function method, we shall show that the solution of problems (1.1)-(1.3) is exponentially decay if the delay parameter $\tau$ is sufficiently small.

We shall use standard notation. By $L^{p}(\Omega)$ we shall denote the space of functions in $\Omega$ whose $p$ th power is integrable, with the norm $\|g\|_{L^{p}}^{p}=\int_{\Omega}|g(x)|^{p} d x, 1 \leq p<+\infty$. The norm in $L^{2}(\Omega)$ we will denote by $\|\cdot\|_{L^{2}}=\|\cdot\|$. By $L^{\infty}(\Omega)$ we denote the space of measurable essentially bounded functions in $\Omega$ with the norm

$$
\|g\|_{L^{\infty}}=\operatorname{ess} \sup _{x \in \Omega}|g(x)| .
$$

For each $\sigma \in \mathbb{R}$ we shall denote by $H^{\sigma}(\Omega)$ the usual Sobolev space of order $\sigma$. By $H_{\mathrm{per}}^{\sigma}(\Omega)$, $\sigma \geq 0$ we shall indicate the space of functions periodic in the sense of (1.2). If $g \in H_{\mathrm{per}}^{\sigma}(\Omega)$ then $g$ has an expansion in a Fourier series,

$$
g(x)=\sum_{k \in \mathbb{Z}} g_{k} \exp (2 k i \pi x)
$$

The norm of $g$ in $H_{\text {per }}^{\sigma}(\Omega)$ will be denoted by

$$
\|g\|_{H^{\sigma}}^{2}=\sum_{k \in \mathbb{Z}}\left(1+|k|^{2}\right)^{\sigma}\left|g_{k}\right|^{2},
$$


which is equivalent to $H^{\sigma}(\Omega)$ norm, $\sigma \geq 0$, according to Temam [16]. We shall denote by $\dot{L}^{2}(\Omega)$ and $\dot{H}^{\sigma}(\Omega)$ the space of functions $g \in L^{2}(\Omega)$ or $H^{\sigma}(\Omega)$ such that

$$
\int_{\Omega} g(x) d x=0
$$

The space $\dot{H}^{\sigma}(\Omega), \sigma \in \mathbb{R}_{+}$, is the space of functions $\left.g \in L^{2}(\Omega)\right)$ such that $g$ satisfies (1.5) and

$$
\sum_{k \in \mathbb{Z}}\left(1+|k|^{2}\right)^{\sigma}\left|g_{k}\right|^{2}<+\infty
$$

In $\dot{H}_{\text {per }}^{1}(\Omega)$ the Poincaré inequality holds, that is, if $g \in \dot{H}_{\text {per }}^{1}(\Omega)$ then

$$
\|g\| \leq C(\Omega)\left\|g^{\prime}\right\| .
$$

The inequality (1.6) shows that $\dot{H}_{\text {per }}^{1}(\Omega)$ is a Hilbert space with scalar product of $H_{0}^{1}(\Omega)$, and $\|u\|_{H^{1}}=(u, u)_{H^{1}}^{1 / 2}$ is a norm on this space equivalent to that induced by $H^{1}(\Omega)$.

The operators $M$ and $L$ of (1.1) are pseudo-differential operators of orders $\mu$ and $s$, respectively, with

$$
\begin{aligned}
& M: \dot{H}_{\mathrm{per}}^{\mu}(\Omega) \rightarrow \dot{L}_{\mathrm{per}}^{2}(\Omega), \quad \mu \geq 1, \mu \in \mathbb{R}, \\
& L: \dot{H}_{\mathrm{per}}^{s}(\Omega) \rightarrow \dot{L}_{\mathrm{per}}^{2}(\Omega), \quad s \geq 0, s \in \mathbb{R},
\end{aligned}
$$

and

$$
\begin{aligned}
& M g(x)=\sum_{k \in \mathbb{Z}} m(k) g_{k} \exp (2 k i \pi x), \\
& L g(x)=\sum_{k \in \mathbb{Z}} l(k) g_{k} \exp (2 k i \pi x)
\end{aligned}
$$

where $m$ and $l$ are the principal symbols of the operators $M$ and $L$, respectively. We assume from now on that the symbols $m$ and $l$ are even functions of $k$ that satisfy the growth conditions:

(i) There exist constants $c_{1}, c_{2}>0$ such that

$$
c_{1}\left(1+|k|^{2}\right)^{\mu} \leq m(k) \leq c_{2}\left(1+|k|^{2}\right)^{\mu} .
$$

(ii) There exist constants $c_{3}, c_{4}>0$ such that

$$
c_{3}|k|^{s} \leq l(k) \leq c_{4}|k|^{s} .
$$

The domains of operators $M$ and $L$ are given by

$$
\begin{aligned}
& D(M)=\left\{g \in \dot{H}_{\mathrm{per}}^{\mu}(\Omega), \sum_{k \in \mathbb{Z}}|m(k)|^{2}\left|g_{k}\right|^{2}<\infty\right\}, \\
& D(L)=\left\{g \in \dot{H}_{\mathrm{per}}^{s}(\Omega), \sum_{k \in \mathbb{Z}}|l(k)|^{2}\left|g_{k}\right|^{2}<\infty\right\} .
\end{aligned}
$$


Let $X$ be a Banach space and $a<b$. We denote by $C^{n}([a, b] ; X)$ the space of $n$ times continuously differentiable functions defined on $[a, b]$ with values in $X$ with the supremum norm and we write $C([a, b] ; X)$ for $C^{0}([a, b] ; X)$.

The main result of this paper is stated as follows.

Theorem 1.1 For any initial condition $u_{0}=u_{0}(x, s) \in C\left([-\tau, 0], \dot{H}_{\mathrm{per}}^{\frac{\mu}{2}}(\Omega)\right), \mu \geq 2, s \geq 0$, and suppose $M$ and L satisfy the assumptions (1.7), (1.8). Then problem (1.1)-(1.3) has a unique global mild solution $u$ on $[-\tau, \infty)$ with

$$
u \in C\left([-\tau,+\infty), \dot{H}_{\mathrm{per}}^{\frac{\mu}{2}}(\Omega)\right) .
$$

Theorem 1.2 Under the assumption of Theorem 1.1, if $\mu=s$, there are $\tau_{0}, \omega, K>0$ such that, for $\tau<\tau_{0}$, the solution of (1.1)-(1.3) satisfies

$$
\left\|M^{1 / 2} u(t)\right\|^{2} \leq \frac{K^{2}}{4} \exp \{-\omega t\}, \quad t \geq 0 .
$$

This paper is organized as follows. In next section, we prove the existence of the solution. Furthermore, we show that the solution is exponentially decay by using the Liapunov function method.

\section{Exponential decay estimates}

Firstly, we briefly show that problem (1.1)-(1.3) is well posed. To conveniences, we denote $\|u\| \leq \lambda_{1}\left\|L^{\frac{1}{2}} u\right\|,\left\|u_{x}\right\| \leq \lambda_{2}\left\|L^{\frac{1}{2}} u\right\|,\|u\| \leq \lambda_{3}\left\|M^{\frac{1}{2}} u\right\|,\left\|u_{x}\right\| \leq \lambda_{4}\left\|M^{\frac{1}{2}} u\right\|,\left\|M^{\frac{1}{2}} u\right\| \leq$ $\lambda_{5}\left\|L^{\frac{1}{2}} u\right\|$.

Proof of Theorem 1.1 By the standard methods as in [9], it is easy to prove that for every initial value $u_{0}=u_{0}(x, s) \in C\left([-\tau, 0], \dot{H}_{\text {per }}^{\frac{\mu}{2}}(\Omega)\right)$, there exists a $T=T\left(u_{0}\right)>0$ such that problem (1.1)-(1.3) has a unique mild solution $u$ on $[-\tau, T]$ with

$$
u(x, t) \in C\left([-\tau, T], \dot{H}_{\mathrm{per}}^{\frac{\mu}{2}}(\Omega)\right)
$$

Furthermore, for any $\tau>0$, the solution of (1.1)-(1.3) does not blow up in finite time. Indeed, multiplying (1.1) by $u$, integrating by parts, we obtain, for $0 \leq t \leq \tau$,

$$
\begin{aligned}
\frac{d}{d t} & \left\|M^{1 / 2} u(t)\right\|^{2}+2 \alpha\left\|L^{1 / 2} u(t)\right\|^{2} \\
& =-2 \int_{0}^{1} u(t) u(t-\tau) u_{x}(t) d x \\
& \leq 2\left\|u_{0}\right\|_{C\left([-\tau, 0], \dot{H}_{\mathrm{per}}^{1}(\Omega)\right)} \int_{0}^{1}\left|u(t) \| u_{x}(t)\right| d x \\
& \leq 2\left\|u_{0}\right\|_{C\left([-\tau, 0], \dot{H}_{\mathrm{per}}^{1}(\Omega)\right)}\|u(t)\|\left\|u_{x}(t)\right\| \\
& \leq 2 \lambda_{1}\left\|L^{1 / 2} u(t)\right\|\left\|u_{0}\right\|_{C\left([-\tau, 0], \dot{H}_{\mathrm{per}}^{1}(\Omega)\right)}\left\|u_{x}(t)\right\| .
\end{aligned}
$$


By the Young inequality, we get

$$
\begin{aligned}
\frac{d}{d t}\left\|M^{1 / 2} u(t)\right\|^{2} & \leq \frac{\lambda_{1}^{2}}{2 \alpha}\left\|u_{0}\right\|_{C\left([-\tau, 0], \dot{H}_{\mathrm{per}}^{\frac{\mu}{2}}(\Omega)\right)}^{2}\left\|u_{x}(t)\right\|^{2} \\
& \leq \frac{\lambda_{1}^{2} \lambda_{4}^{2}}{2 \alpha}\left\|u_{0}\right\|_{C\left([-\tau, 0], \dot{H}_{\mathrm{per}}^{2}(\Omega)\right)}^{2}\left\|M^{1 / 2} u(t)\right\|^{2},
\end{aligned}
$$

which implies that

$$
\left\|M^{1 / 2} u(t)\right\|^{2} \leq K_{1}\left(\left\|u_{0}\right\|_{C\left([-\tau, 0], \dot{H}_{\text {per }}^{2}(\Omega)\right)}\right)
$$

where $K_{1}\left(\left\|u_{0}\right\|_{C\left([-\tau, 0], \dot{H}_{\text {per }}^{\frac{\mu}{2}}(\Omega)\right)}\right)$ is a positive constant depending on $\left\|u_{0}\right\|_{C\left([-\tau, 0], \dot{H}_{\text {per }}^{\frac{\mu}{2}}(\Omega)\right)} . \operatorname{Re-}$ peating the above procedure, we can prove that, for $n \tau \leq t \leq(n+1) \tau(n=1,2, \ldots)$,

$$
\left\|M^{1 / 2} u(t)\right\|^{2} \leq K_{1}\left(n,\left\|u_{0}\right\|_{C\left([-\tau, 0], \dot{H}_{\mathrm{per}}^{2}(\Omega)\right)}^{\mu}\right) .
$$

In summary, we have proved Theorem 1.1.

Secondly, we prove that our main result about the exponential stability. To this end, we introduce the following notations. For a given initial condition $u_{0}=u_{0}(x, s) \in C([-\tau, 0]$, $\left.\dot{H}_{\text {per }}^{\frac{\mu}{2}}(\Omega)\right)$, denote

$$
K=\sup _{-\tau \leq s \leq 0}\left\|M^{1 / 2} u_{0}(s)\right\|+\sqrt{8\left\|M^{1 / 2} u_{0}(0)\right\|^{2}}
$$

Set

$$
\sigma=\sup \left\{\delta>0:\left\|M^{1 / 2} u_{0}(0)\right\|^{2} \leq \frac{K^{2}}{4}, 0 \leq \tau \leq \delta\right\}
$$

and let $\tau_{0}$ be small enough, such that for any $\tau, 0 \leq \tau<\tau_{0} \leq \sigma$,

$$
\omega=\frac{2 \alpha}{\lambda_{5}^{2}}-\frac{2 \lambda_{2}^{2} K}{\lambda_{5}^{2}} \sqrt{\frac{\tau\left[\alpha+\frac{\lambda_{4}^{4} K^{2}}{\varepsilon} \tau\right]}{2 \lambda_{3}^{-2}-\varepsilon}}>0 .
$$

Proof of Theorem 1.2 Let

$$
T_{0}=\sup \left\{\delta:\left\|M^{1 / 2} u(t)\right\|^{2} \leq K^{2}, 0 \leq t \leq \delta\right\} .
$$

Since

$$
\left\|M^{1 / 2} u(0)\right\|^{2} \leq K^{2},
$$

and $\left\|M^{1 / 2} u(t)\right\|$ is continuous, we have $T_{0}>0$. We shall prove that $T_{0}=+\infty$. For this, we argue by contradiction. If $T_{0}<+\infty$, then we have

$$
\left\|M^{1 / 2} u(t)\right\|^{2} \leq K^{2}, \quad \forall-\tau \leq t \leq T_{0}
$$


and

$$
\left\|M^{1 / 2} u\left(T_{0}\right)\right\|^{2}=K^{2}
$$

Multiplying (1.1) by $u$, then integrating on $\Omega$ with respect to $x$, we obtain

$$
\frac{d}{d t}\left\|M^{1 / 2} u(t)\right\|^{2}+2 \alpha\left\|L^{1 / 2} u(t)\right\|^{2}=-2 \int_{0}^{1} u(t) u(t-\tau) u_{x}(t) d x
$$

Since $\int_{0}^{1} u^{2}(t) u_{x}(t) d x=0$, we have

$$
\frac{d}{d t}\left\|M^{1 / 2} u(t)\right\|^{2}+2 \alpha\left\|L^{1 / 2} u(t)\right\|^{2}=\Phi
$$

where

$$
\Phi=-2 \int_{0}^{1} u(t)[u(t-\tau)-u(t)] u_{x}(t) d x
$$

We now majorize $\Phi$ in the right hand side of (2.7). Firstly, since

$$
|u(x, t)| \leq\left\|u_{x}(x, t)\right\|, \quad 0 \leq x \leq 1,
$$

we have

$$
\begin{aligned}
\Phi & \leq 2 \int_{0}^{1}|u(t-\tau)-u(t)||u(t)|\left|u_{x}(t)\right| d x \\
& \leq 2 \int_{0}^{1}|u(t-\tau)-u(t)|\left\|u_{x}(t)\right\|\left|u_{x}(t)\right| d x \\
& \leq 2\left\|u_{x}(t)\right\| \int_{0}^{1}|u(t-\tau)-u(t)|\left|u_{x}(t)\right| d x \\
& \leq 2\left\|u_{x}(t)\right\|\left(\int_{0}^{1}|u(t-\tau)-u(t)|^{2} d x\right)^{\frac{1}{2}}\left(\int_{0}^{1}\left|u_{x}(t)\right|^{2} d x\right)^{\frac{1}{2}} \\
& =2\left\|u_{x}(t)\right\|^{2}\left(\int_{0}^{1}|u(t-\tau)-u(t)|^{2} d x\right)^{\frac{1}{2}} \\
& =2\left\|u_{x}(t)\right\|^{2}\left(\int_{0}^{1}\left|\int_{t-\tau}^{t} u_{s}(s) d s\right|^{2} d x\right)^{\frac{1}{2}} \\
& \leq 2\left\|u_{x}(t)\right\|^{2}\left(\int_{0}^{1}\left(\int_{t-\tau}^{t} u_{s}^{2}(s) d s \int_{t-\tau}^{t} d s\right)^{\frac{1}{2}} d x\right)^{\frac{1}{2}} \\
& =2 \sqrt{\tau}\left\|u_{x}(t)\right\|^{2}\left(\int_{0}^{1} \int_{t-\tau}^{t} u_{s}^{2}(s) d s d x\right)^{\frac{1}{2}} .
\end{aligned}
$$

Let

$$
\Psi=\left(\int_{0}^{1} \int_{t-\tau}^{t} u_{s}^{2}(s) d s d x\right)^{\frac{1}{2}}
$$


we have

$$
\Phi \leq 2 \sqrt{\tau}\left\|u_{x}(t)\right\|^{2} \Psi
$$

and

$$
\Psi^{2} \leq \lambda_{3}^{2} \int_{t-\tau}^{t}\left\|M^{1 / 2} u_{s}(s)\right\|^{2} d s
$$

We now want to estimate $\Psi$. To this end, multiplying (1.1) by $u_{t}$, then integrating on $\Omega$ with respect to $x$, we obtain

$$
\left\|M^{1 / 2} u_{t}(t)\right\|^{2}+\frac{\alpha}{2} \frac{d}{d t}\left\|L^{1 / 2} u(t)\right\|^{2}+\int_{0}^{1} u_{t}(t) u(t-\tau) u_{x}(t) d x=0 .
$$

Integrating on $[t-\tau, t], 0 \leq t \leq T_{0}$, we obtain

$$
2 \int_{t-\tau}^{t}\left\|M^{1 / 2} u_{s}(s)\right\|^{2} d s+\alpha\left\|L^{1 / 2} u(t)\right\|^{2}-\alpha\left\|L^{1 / 2} u(t-\tau)\right\|^{2}+H=0,
$$

where

$$
H=2 \int_{t-\tau}^{t} \int_{0}^{1} u_{s}(s) u(s-\tau) u_{x}(s) d x d s,
$$

which implies that

$$
2 \int_{t-\tau}^{t}\left\|M^{1 / 2} u_{s}(s)\right\|^{2} d s \leq \alpha\left\|L^{1 / 2} u(t-\tau)\right\|^{2}-H .
$$

We now majorize $H$ :

$$
\begin{aligned}
H & \leq 2 \int_{t-\tau}^{t} \int_{0}^{1}\left|u_{s}(s)\right|\left|u(s-\tau) \| u_{x}(s)\right| d x d s \\
& \leq 2 \int_{t-\tau}^{t} \int_{0}^{1}\left\|u_{x}(s-\tau)\right\|\left(\left|u_{s}(s)\right|\left|u_{x}(s)\right|\right) d x d s \\
& \leq 2 \lambda_{4} K \int_{t-\tau}^{t} \int_{0}^{1}\left|u_{s}(s) \| u_{x}(s)\right| d x d s \\
& \leq 2 \lambda_{4} K\left(\int_{t-\tau}^{t} \int_{0}^{1} u_{s}^{2}(s) d x d s\right)^{\frac{1}{2}}\left(\int_{t-\tau}^{t} \int_{0}^{1} u_{x}^{2}(s) d x d s\right)^{\frac{1}{2}} \\
& \leq \varepsilon \Psi^{2}+\frac{\lambda_{4}^{2} K^{2}}{\varepsilon} \int_{t-\tau}^{t} \int_{0}^{1} u_{x}^{2}(s) d x d s \\
& \leq \varepsilon \Psi^{2}+\frac{\lambda_{4}^{2} K^{2}}{\varepsilon} \int_{t-\tau}^{t} K^{2} d s \\
& \leq \varepsilon \Psi^{2}+\frac{\lambda_{4}^{2} K^{4}}{\varepsilon} \tau,
\end{aligned}
$$

with $0<\varepsilon<2 \lambda_{3}^{-2}$. Thus by (2.10)-(2.11), we have

$$
2 \int_{t-\tau}^{t}\left\|M^{1 / 2} u_{s}(s)\right\|^{2} d s \leq \alpha K^{2}+\varepsilon \Psi^{2}+\frac{\lambda_{4}^{2} K^{4}}{\varepsilon} \tau,
$$


furthermore, by (2.9), we get

$$
\Psi \leq K \sqrt{\frac{\left[\alpha+\frac{\lambda_{4}^{4} K^{2}}{\varepsilon} \tau\right]}{2 \lambda_{3}^{-2}-\varepsilon}}, \quad \forall 0 \leq t \leq T_{0} .
$$

Then (2.8) and (2.13) implies that

$$
\begin{aligned}
\Phi & \leq 2 \lambda_{2}^{2} K \sqrt{\frac{\tau\left[\alpha+\frac{\lambda_{4}^{4} K^{2}}{\varepsilon} \tau\right]}{2 \lambda_{3}^{-2}-\varepsilon}}\left\|u_{x}(t)\right\|^{2} \\
& \leq 2 \lambda_{2}^{2} K \sqrt{\frac{\tau\left[\alpha+\frac{\lambda_{4}^{4} K^{2}}{\varepsilon} \tau\right]}{2 \lambda_{3}^{-2}-\varepsilon}}\left\|L^{1 / 2} u(t)\right\|^{2} .
\end{aligned}
$$

Thus, by (2.7) and (2.14), we obtain

$$
\frac{d}{d t}\left\|M^{1 / 2} u(t)\right\|^{2}+\left\|L^{1 / 2} u(t)\right\|^{2}\left(2 \alpha-2 \lambda_{2}^{2} K \sqrt{\frac{\tau\left[\alpha+\frac{\lambda_{4}^{4} K^{2}}{\varepsilon} \tau\right]}{2 \lambda_{3}^{-2}-\varepsilon}}\right) \leq 0
$$

furthermore,

$$
\frac{d}{d t}\left\|M^{1 / 2} u(t)\right\|^{2}+\omega\left\|M^{1 / 2} u(t)\right\|^{2} \leq 0,
$$

where $\omega$ is defined by (2.3). Solving the above inequality gives

$$
\left\|M^{1 / 2} u(t)\right\|^{2} \leq\left\|M^{1 / 2} u_{0}(x, 0)\right\|^{2} e^{-\omega t} \leq \frac{K^{2}}{4} e^{-\omega t}, \quad 0 \leq t \leq T_{0} .
$$

Hence

$$
\left\|M^{1 / 2} u(t)\left(T_{0}\right)\right\|^{2} \leq K^{2} e^{-\omega T_{0}},
$$

which is in contradiction with (2.6). Therefore, we have proved that $T_{0}=+\infty$ and then (1.9) follows from (2.16). Thus we have completed the proof of Theorem 1.2.

\section{Competing interests}

The authors declare that they have no competing interests.

Authors' contributions

FW gave access to information, PZ formulated the outline and ZC wrote the essay. All authors read and approved the final manuscript.

\section{Author details}

${ }^{1}$ College of Mathematics and Information Science, Neijiang Normal University, Neijiang, Sichuan 641100, China. ${ }^{2}$ College of Mathematics and Software Science, Sichuan Normal University, Chengdu, 610066, China. ${ }^{3}$ School of Mathematics and Statistics, Southwest University, Chongqing, 400715, China.

\section{Acknowledgements}

This work was supported by the Postdoctor Research Fund of Chongqing (No. YuXM201102006), the National Natural Science Foundation of China (No. 61273020), and the Fundamental Research Funds for the Central Universities (No. XDJK2009C070).

Received: 6 January 2015 Accepted: 3 July 2015 Published online: 01 August 2015 


\section{References}

1. Benjamin, TB, Bona, JL, Mahony, JJ: Model equations for long waves in nonlinear dispersive systems. Philos. Trans. R. Soc. Lond. 272, 47-78 (1972)

2. Amick, CJ, Bona, JL, Schonbek, ME: Decay of solutions of some nonlinear wave equations. J. Differ. Equ. 81, 1-49 (1989)

3. Wang, B, Yang, W: Finite-dimensional behavior for the Benjamin-Bona-Mahony equation. J. Phys. A, Math. Gen. 30 4877-4885 (1997)

4. Bona, J, Luo, L: Decay of solutions to nonlinear, dispersive wave equations. Differ. Integral Equ. 6(5), $961-980$ (1993)

5. Zhang, L: Decay of solution of generalized Benjamin-Bona-Mahony-Burgers equations in $n$-space dimensions. Nonlinear Anal. 25(12), 1343-1369 (1995)

6. Biller, P: Large-time behaviour of periodic solutions to dissipative equations of Korteweg-de Vries-Burgers type. Bull. Pol. Acad. Sci., Math. 32(7-8), 401-405 (1984)

7. Bisognin, V, Menzala, GP: Decay rates of the solutions of nonlinear dispersive equations. Proc. R. Soc. Edinb. A 124 1231-1246 (1994)

8. Bisognin, V, Menzala, GP: Asymptotic behaviour of nonlinear dispersive models with variable coefficients. Ann. Mat. Pura Appl. (4) CLXVIII, 219-235 (1995)

9. Astaburuaga, MA, Bisognin, E, Bisognin, V, Fernandez, C: Global attractor and finite dimensionality for a class dissipative equations of BBM's type. Electron. J. Differ. Equ. 1998, 25 (1998)

10. Friesecke, G: Convergence to equilibrium for delay-diffusion equations with small delay. J. Dyn. Differ. Equ. 5, 89-103 (1993)

11. Luckhaus, S: Global boundedness for a delay differential equation. Trans. Am. Math. Soc. 294, 767-774 (1986)

12. Oliva, SM: Reaction-diffusion equations with nonlinear boundary delay. J. Dyn. Differ. Equ. 11, $279-296$ (1999)

13. Liu, W: Asymptotic behavior of solutions of time-delayed Burgers equation. Discrete Contin. Dyn. Syst., Ser. B 2(1), 47-56 (2002)

14. Zhu, CS, Mu, CL: Exponential decay estimates for time-delayed Benjamin-Bona-Mahony equations. Appl. Anal. 87, 401-408 (2008)

15. Zhu, CS: Asymptotic behavior of solutions for the time-delayed Kuramoto-Sivashinsky equation. Z. Anal. Anwend. 33 429-439 (2014)

16. Temam, R: Infinite Dimensional Dynamical Systems in Mechanics and Physics, 2nd edn. Springer, Berlin (1997)

\section{Submit your manuscript to a SpringerOpen ${ }^{\ominus}$ journal and benefit from:}

- Convenient online submission

- Rigorous peer review

- Immediate publication on acceptance

- Open access: articles freely available online

High visibility within the field

Retaining the copyright to your article 